\title{
Solving the Inter-Ring Distances Optimization Problem for Pentapolar and Sextopolar Concentric Ring Electrodes Based on the Negligible Dimensions Model of the Electrode ${ }^{\dagger}$
}

\author{
Oleksandr Makeyev*(D), Alana Lee and Ashton Begay
}

check for updates

Citation: Makeyev, O.; Lee, A.;

Begay, A. Solving the Inter-Ring Distances Optimization Problem for Pentapolar and Sextopolar Concentric Ring Electrodes Based on the Negligible Dimensions Model of the Electrode. Eng. Proc. 2021, 10, 8. https: / / doi.org/10.3390/ ecsa-8-11280

Academic Editor: Alberto Vallan

Published: 1 November 2021

Publisher's Note: MDPI stays neutral with regard to jurisdictional claims in published maps and institutional affiliations.

Copyright: (c) 2021 by the authors. Licensee MDPI, Basel, Switzerland. This article is an open access article distributed under the terms and conditions of the Creative Commons Attribution (CC BY) license (https:/ / creativecommons.org/licenses/by/ $4.0 /)$.

\author{
School of STEM, Diné College, Tsaile, AZ 86556, USA; alanalee@dinecollege.edu (A.L.); \\ aryanbegay@dinecollege.edu (A.B.) \\ * Correspondence: omakeyev@dinecollege.edu; Tel.: +1-928-724-6960 \\ + Presented at the 8th International Electronic Conference on Sensors and Applications, 1-15 November 2021; \\ Available online: https:/ / ecsa-8.sciforum.net.
}

\begin{abstract}
Concentric ring electrodes are noninvasive and wearable sensors for electrophysiological measurement capable of estimating the surface Laplacian (second spatial derivative of surface potential) at each electrode. Previously, progress was made toward optimization of inter-ring distances (distances between the recording surfaces of a concentric ring electrode), maximizing the accuracy of the surface Laplacian estimate based on the negligible dimensions model of the electrode. However, this progress was limited to tripolar (number of concentric rings $n$ equal to 2 ) and quadripolar ( $n=3$ ) electrode configurations only. In this study, the inter-ring distances optimization problem is solved for pentapolar $(n=4)$ and sextopolar $(n=5)$ concentric ring electrode configurations using a wide range of truncation error percentiles ranging from 1st to 25th. Obtained results also suggest consistency between all the considered concentric ring electrode configurations corresponding to $n$ ranging from 2 to 5 that may allow estimation of optimal ranges of inter-ring distances for electrode configurations with $n \geq 6$. Therefore, this study may inform future concentric ring electrode design for $n \geq 4$ which is important since the accuracy of surface Laplacian estimation has been shown to increase with an increase in $n$.
\end{abstract}

Keywords: electrophysiology; measurement; wearable sensors; noninvasive; concentric ring electrodes; Laplacian; estimation; optimization; inter-ring distances; negligible dimensions model

\section{Introduction}

Concentric ring electrodes (CREs; tripolar configuration shown in Figure 1A) are noninvasive and wearable sensors for electrophysiological measurement capable of estimating the surface Laplacian (second spatial derivative of surface potential) at each electrode, which is not feasible with conventional disc electrodes (Figure 1B), and constitutes CRE's practical relevance to the wearable sensor field [1-10]. Recent examples of biomedical applications utilizing CREs involve electroencephalograms (source localization of highfrequency activity [5] and seizure detection [8] in epilepsy patients), electroenterograms (identification of the intestinal slow waves [2]), and electromyograms (evaluation of swallowing [11] and respiratory [10] muscle activity). Previously, progress was made toward optimization of inter-ring distances (distances between the recording surfaces of a CRE), maximizing the accuracy of the surface Laplacian estimate based on the negligible dimensions model (NDM) of the electrode [12]. In NDM, a single point of negligible diameter represents the central disc surrounded by concentric circles of negligible width that represent the concentric rings. In [12], the inter-ring distances optimization problem was solved for tripolar (number of concentric rings $n$ equal to 2) and quadripolar $(n=3) \mathrm{CRE}$ configurations and 5th and 10th percentiles of absolute value of truncation term coefficient for the lowest remaining term order since this coefficient has been shown to be a predictor 
of the Laplacian estimation error $[12,13]$. Obtained results have been validated using finite element method modeling [12].



Figure 1. Tripolar concentric ring electrode (A) and conventional disc electrode (B).

In this study, the NDM-based inter-ring distances optimization problem is solved for pentapolar (PCRE; Figure 2A; $n=4$ ) and sextopolar (SCRE; Figure 2B; $n=5$ ) CRE configurations while also extending the range of percentiles compared to [12] for all the CRE configurations optimized so far. Specifically, the following steps have been taken: first, truncation term coefficient functions have been derived for the two aforementioned CRE configurations. Second, absolute values of truncation term coefficients have been computed for an extensive grid of possible combinations of values of function arguments. Third, thresholds corresponding to percentiles ranging from 1st to 25th have been calculated. Finally, these threshold values were used to determine the ranges of optimized inter-ring distances for each CRE configuration via determination and model fitting of a boundary hyperplane. Extension of the percentile range from [12] is related to the question of how small can the distances between the recording surfaces become without partial shorting due to salt bridges becoming a significant factor affecting the accuracy of Laplacian estimation. This question is still to be answered in the future using physical CRE prototypes. If prototype assessment results would suggest that physical considerations render the interring distances within, for example, the 5th percentile region impractical, then inter-ring distances within the higher percentile region will be used, such as, for example, the 10th percentile region (which was the only other percentile value considered in [12]) or higher. However, if prototype assessment results would suggest otherwise, then using even lower percentile values such as the 1 st or the 3 rd percentile could be feasible. To accommodate the range of possible scenarios, this study utilizes the wide 1st to 25th percentile range not just for PCRE and SCRE cases but also to extend the results of [12] for tripolar and quadripolar CRE configurations.



Figure 2. Negligible dimensions models of pentapolar (A) and sextopolar (B) concentric ring electrode configurations.

\section{Methods}

2.1. Defining the Inter-Ring Distances Optimization Problem for PCRE and SCRE Configurations

Following the methodology established in [12], the main steps taken to define the NDM-based inter-ring distances optimization problem for the PCRE configuration (Figure 2A) with the first middle ring radius $\alpha r$, the second middle ring radius $\beta r$, the third middle ring radius $\gamma r$, and the outer ring radius $r$ to determine the optimal range of values for coefficients $\alpha, \beta$, and $\gamma$ such that $0<\alpha<\beta<\gamma<1$ are listed below. Identical steps were taken for the SCRE configuration with an additional middle ring of radius $\delta r$ such that $0<\alpha<\beta<\gamma<\delta<1$. More detail on each step is available in [12] for tripolar and quadripolar CRE configurations. First, for each of the four rings, the integral of the Taylor series has been taken along the circle of the corresponding radius. Second, the matrix 
of truncation term coefficients $A^{P C R E}$ was determined in Equation (1). Respective matrix $A^{S C R E}$ for SCRE configuration is given in Equation (2). Third, the null space of $A^{P C R E}$ was determined up to a (multiplication by a) constant factor. Fourth, four Taylor series integrals were combined into a weighted linear combination solved for the Laplacian using the null space vector as coefficients and allowing cancellation of 4 th, 6 th, and 8 th order truncation terms (as shown in [14], CRE with $n$ rings allows cancellation of truncation terms up to the order of $2 n$, which for the case of PCRE configuration corresponds to the 8th order). Fifth, truncation term coefficient function $c^{P C R E}(\alpha, \beta, \gamma, k)$ was derived for even truncation term order $k \geq 10$ in Equation (3). Respective SCRE configuration function $c^{S C R E}(\alpha, \beta$, $\gamma, \delta, k)$ derived for even truncation term order $k \geq 12$ is given in Equation (4). Sixth, a constrained optimization problem was defined to minimize absolute values of truncation term coefficients, thus allowing minimization of the truncation error and maximization of the accuracy of surface Laplacian estimation. Seventh, this inter-ring distances optimization problem has been solved for the lowest remaining truncation term order equal to 10 (since it is the main contributor to the truncation error [15]) using a wide range of percentile values (1st, 3rd, 5th, 10th, 15th, 20th, and 25th): $\min _{0<\alpha<\beta<\gamma<1}\left|c^{P C R E}(\alpha, \beta, \gamma, 10)\right|$. The same steps (and percentile values) were applied to solve the inter-ring distances optimization problem for the SCRE configuration: $\min _{0<\alpha<\beta<\gamma<\delta<1}\left|c^{S C R E}(\alpha, \beta, \gamma, \delta, 12)\right|$.

\subsection{Solving the Inter-Ring Distances Optimization Problem for PCRE and SCRE Configurations}

Solving the inter-ring distances problem for PCRE and SCRE configurations following the approach from [12] involves using a wide range of truncation error percentiles to identify points on the boundary hyperplane. For the PCRE configuration, absolute values of truncation term coefficients based on function $c^{P C R E}(\alpha, \beta, \gamma, k)$ from Equation (3) were computed for all the combinations of values of $0<\alpha<\beta<\gamma<1$ with the increment of $1 \%$ (or 0.01 ) and $k=10$. For the SCRE configuration, function $c^{S C R E}(\alpha, \beta, \gamma, \delta, k)$ from Equation (4) was computed for all the combinations of values of $0<\alpha<\beta<\gamma<\delta<1$ with the same increment of $1 \%$ and $k=12$. Percentiles were used to find the values of $\alpha, \beta$, and $\gamma$ (for PCRE configuration) and $\alpha, \beta, \gamma$, and $\delta$ (for SCRE configuration) that corresponded to points on the boundary hyperplane separating the absolute values of the 10th order (for PCRE) and of the 12th order (for SCRE) truncation term coefficients below and above the specific percentile. The resulting boundary hyperplane points were fitted with a nonlinear regression model of the form $\alpha \beta \gamma=m$ for PCRE and $\alpha \beta \gamma \delta=m$ for SCRE, respectively, where $m$ is a real constant. The choice of these particular models stemmed from their consistency with the models used for tripolar and quadripolar CREs in [12] which is discussed in the Section 4 of this paper.

$$
\begin{gathered}
A^{\text {PCRE }}=\left(\begin{array}{cccc}
\alpha^{4} & \beta^{4} & \gamma^{4} & 1^{4} \\
\alpha^{6} & \beta^{6} & \gamma^{6} & 1^{6} \\
\alpha^{8} & \beta^{8} & \gamma^{8} & 1^{8}
\end{array}\right)=\left(\begin{array}{llll}
\alpha^{4} & \beta^{4} & \gamma^{4} & 1 \\
\alpha^{6} & \beta^{6} & \gamma^{6} & 1 \\
\alpha^{8} & \beta^{8} & \gamma^{8} & 1
\end{array}\right), \\
A^{\text {SCRE }}=\left(\begin{array}{ccccc}
\alpha^{4} & \beta^{4} & \gamma^{4} & \delta^{4} & 1 \\
\alpha^{6} & \beta^{6} & \gamma^{6} & \delta^{6} & 1 \\
\alpha^{8} & \beta^{8} & \gamma^{8} & \delta^{8} & 1 \\
\alpha^{10} & \beta^{10} & \gamma^{10} & \delta^{10} & 1
\end{array}\right),
\end{gathered}
$$

$$
\begin{aligned}
& c^{P C R E}(\alpha, \beta, \gamma, k)=-\frac{4 \alpha^{-2} \beta^{-2} \gamma^{-2}}{\left(\alpha^{2}-1\right)\left(\alpha^{2}-\beta^{2}\right)\left(\beta^{2}-1\right)\left(\alpha^{2}-\gamma^{2}\right)\left(\beta^{2}-\gamma^{2}\right)\left(\gamma^{2}-1\right)} \\
& \times\left[\begin{array}{l}
\alpha^{k} \beta^{4}\left(\beta^{2}-1\right) \gamma^{4}\left(\beta^{2}-\gamma^{2}\right)\left(\gamma^{2}-1\right)+\alpha^{8}\left(\beta^{k}\left(\gamma^{4}-\gamma^{6}\right)+\beta^{4}\left(\gamma^{6}-\gamma^{k}\right)+\beta^{6}\left(\gamma^{k}-\gamma^{4}\right)\right)+ \\
+\alpha^{4}\left(\beta^{k}\left(\gamma^{6}-\gamma^{8}\right)+\beta^{6}\left(\gamma^{8}-\gamma^{k}\right)+\beta^{8}\left(\gamma^{k}-\gamma^{6}\right)\right)+\alpha^{6}\left(\beta^{k} \gamma^{4}\left(\gamma^{4}-1\right)+\beta^{8}\left(\gamma^{4}-\gamma^{k}\right)+\beta^{4}\left(\gamma^{k}-\gamma^{8}\right)\right)
\end{array}\right],
\end{aligned}
$$




$$
\begin{aligned}
& c^{S C R E}(\alpha, \beta, \gamma, \delta, k)=\frac{4 \alpha^{2} \beta^{2} \delta^{2} \gamma^{2}}{\left(\alpha^{2}-1\right)\left(\beta^{2}-1\right)\left(\delta^{2}-1\right)\left(\gamma^{2}-1\right)} \\
& \times\left[\begin{array}{c}
1+\frac{\alpha^{k-4}\left(\beta^{2}-1\right)\left(\delta^{2}-1\right)\left(\gamma^{2}-1\right)}{\left(\alpha^{2}-\beta^{2}\right)\left(\alpha^{2}-\delta^{2}\right)\left(\alpha^{2}-\gamma^{2}\right)}-\frac{\left(\alpha^{2}-1\right) \beta^{k-4}\left(\delta^{2}-1\right)\left(\gamma^{2}-1\right)}{\left(\alpha^{2}-\beta^{2}\right)\left(\beta^{2}-\delta^{2}\right)\left(\beta^{2}-\gamma^{2}\right)}+ \\
+\frac{\left(\alpha^{2}-1\right)\left(\beta^{2}-1\right) \delta^{k-4}\left(\gamma^{2}-1\right)}{\left(\alpha^{2}-\delta^{2}\right)\left(\beta^{2}-\delta^{2}\right)\left(\delta^{2}-\gamma^{2}\right)}-\frac{\left(\alpha^{2}-1\right)\left(\beta^{2}-1\right)\left(\delta^{2}-1\right) \gamma^{k-4}}{\left(\alpha^{2}-\gamma^{2}\right)\left(\gamma^{2}-\beta^{2}\right)\left(\gamma^{2}-\delta^{2}\right)}
\end{array}\right] .
\end{aligned}
$$

\section{Results}

Obtained results for CRE configurations including PCRE and SCRE as well as an extension of results for tripolar (no curve or hyperplane fitting necessary) and quadripolar (nonlinear boundary fitted with the rectangular hyperbola curve $\alpha \beta=m$ ) CREs to the same range of percentiles are presented in Table 1 . Table 1 also includes the respective values of the goodness-of-fit metric R-squared $\left(R^{2}\right)$ that represents the percentage of the total variation in the data explained by the model fit for all the CRE configurations except for the tripolar one. The plot of the fitted boundary hyperplane model separating the absolute values of the 10th order truncation term coefficients below and above the 5th percentile for the PCRE configuration is presented in Figure 3. The goodness-of-fit metric $\mathrm{R}^{2}$ for the fitted model in Figure 3 was equal to 0.995 or $99.5 \%$ (Table 1).

Table 1. Values of the model fitting constant $(m)$ and goodness-of-fit metric R-squared $\left(\mathrm{R}^{2}\right)$ for a range of CRE configurations and percentile values.

\begin{tabular}{ccccccccc}
\hline \multirow{2}{*}{ Percentile } & \multicolumn{2}{c}{ Tripolar CRE } & \multicolumn{2}{c}{ Quadripolar CRE } & \multicolumn{2}{c}{ PCRE } & \multicolumn{2}{c}{ SCRE } \\
\cline { 2 - 9 } & $\boldsymbol{m}$ & $\mathbf{R}^{\mathbf{2}}$ & $\boldsymbol{m}$ & $\mathbf{R}^{\mathbf{2}}$ & $\boldsymbol{m}$ & $\mathbf{R}^{\mathbf{2}}$ & $\boldsymbol{m}$ & $\mathbf{R}^{\mathbf{2}}$ \\
\hline 1st & 0.098 & - & 0.094 & 0.997 & 0.096 & 0.989 & 0.092 & 0.986 \\
\hline 3rd & 0.171 & - & 0.166 & 0.998 & 0.165 & 0.994 & 0.158 & 0.991 \\
\hline 5th & $0.221^{1}$ & - & $0.214^{1}$ & $0.998^{1}$ & 0.213 & 0.995 & 0.204 & 0.992 \\
\hline 10th & $0.313^{1}$ & - & $0.303^{1}$ & 0.998 & 0.3 & 0.996 & 0.288 & 0.991 \\
\hline 15th & 0.383 & - & 0.372 & 0.998 & 0.367 & 0.995 & 0.352 & 0.99 \\
\hline 20th & 0.442 & - & 0.43 & 0.998 & 0.424 & 0.995 & 0.407 & 0.989 \\
\hline 25th & 0.494 & - & 0.481 & 0.998 & 0.474 & 0.994 & 0.455 & 0.987 \\
\hline
\end{tabular}

${ }^{1}$ results reported in [12].

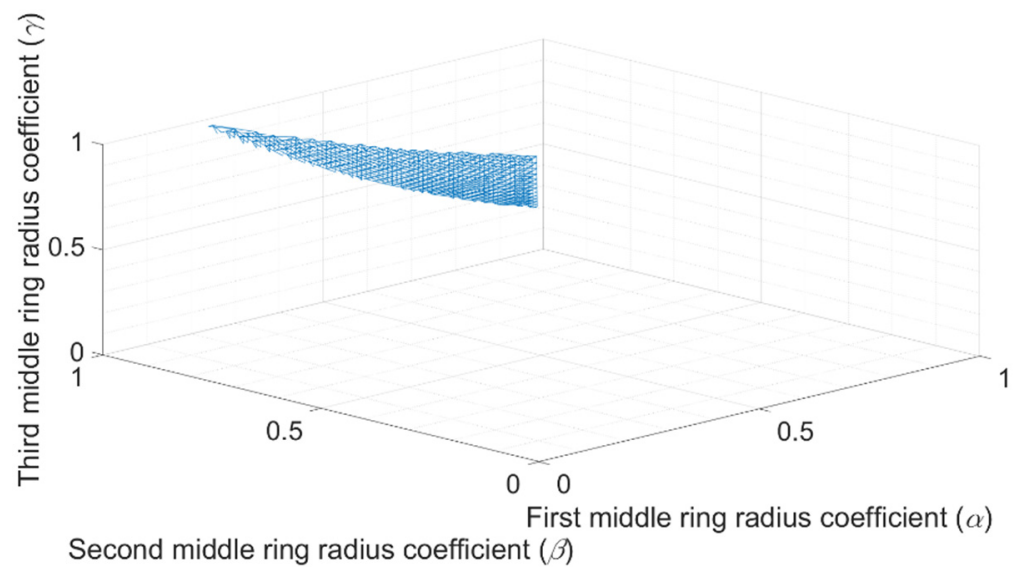

Figure 3. Fitted boundary hyperplane model $\left(m=0.213 ; \mathrm{R}^{2}=0.995\right)$ separating the absolute values of the 10th order truncation term coefficients below and above the 5 th percentile for the PCRE configuration.

\section{Discussion}

This study demonstrates that the NDM-based inter-ring distances optimization approach from [12] can be extended from tripolar and quadripolar CRE configurations to PCREs and SCREs. Obtained results are presented in Table 1 and can be interpreted easily 
for a specific percentile value. For example, for the 5 th percentile, optimal ranges of values of $\alpha, \beta$, and $\gamma$ (for PCRE configuration) and $\alpha, \beta, \gamma$, and $\delta$ (for SCRE configuration) are determined by inequalities $0<\alpha<\beta<\gamma<1$ and $\alpha \beta \gamma \leq 0.213$ and by inequalities $0<\alpha<\beta$ $<\gamma<\delta<1$ and $\alpha \beta \gamma \delta \leq 0.204$, respectively. Moreover, this inter-ring distances optimization approach can be extended further to any larger number of concentric rings $n$ even though the number of decision variables increases by one for each additional concentric ring (Figure 2), further increasing the complexity of the optimization problem. For example, solving it for the septapolar $(n=6)$ CRE configuration results in the total number of values of the 14th order truncation term coefficient function computed with the same increment of $1 \%$ used in this study equal to $71,457,453$. This is compared to the 156,830 values of $c^{P C R E}$ $(\alpha, \beta, \gamma, 10)$ and $3,762,786$ values of $c^{S C R E}(\alpha, \beta, \gamma, \delta, 12)$ functions used in this study. Determining the points on the boundary hyperplane, which requires checking every function value for falling below the specific percentile while at least one of the surrounding values within the $1 \%$ increment along the $\alpha, \beta, \gamma, \delta$, and $\varepsilon$ (for septapolar CRE) axes falls above this percentile, becomes computationally intensive. Fortunately, the result described below provides an opportunity to roughly estimate optimal ranges for larger values of $n$.

Another important result of this study is highlighting the consistency between fitted models with high goodness-of-fit ( $R^{2}$ in Table 1$)$ as well as their constants ( $m$ in Table 1$)$ for CRE configurations with 2 to 5 concentric rings. Specifically, fitted models range from $\alpha \leq m$ (tripolar CRE) to $\alpha \beta \leq m$ (quadripolar CRE) to $\alpha \beta \gamma \leq m$ (PCRE) to $\alpha \beta \gamma \delta \leq m$ (SCRE). It is not unreasonable to hypothesize that for the septapolar CRE configuration with an additional middle concentric ring of radius $\varepsilon r$ such that $0<\alpha<\beta<\gamma<\delta<\varepsilon<1$ the fitted model with high goodness-of-fit would be $\alpha \beta \gamma \delta \varepsilon \leq m$, etc. Moreover, even values of constant $m$ for the aforementioned fitted models are consistent for a specific percentile. For example, for the 5th percentile, $m$ ranges from 0.221 (tripolar CRE) to 0.214 (quadripolar CRE) to 0.213 (PCRE) to 0.204 (SCRE). It is not unreasonable to hypothesize that for the septapolar CRE configuration, the value of $m$ will be less than or equal to 0.204 . It should also be noted that in Table 1 values of $\mathrm{R}^{2}$ are decreasing for each percentile value with an increase in $n$ while still remaining high ( $>0.985)$ overall. For example, for the same 5 th percentile, the value of $R^{2}$ decreases from 0.998 (quadripolar CRE) to 0.995 (PCRE) to 0.992 (SCRE).

\section{Conclusions}

Ability to accurately estimate the surface Laplacian at each electrode constitutes the primary biomedical significance of CREs and this study allows maximizing estimation accuracy for two additional electrode configurations with larger numbers (4 and 5) of concentric rings $n$ which is important since it has been shown that accuracy of Laplacian estimation via CREs increases with an increase in $n$. Other contributions to the technical novelty of this study include extension of the previous optimization results for CRE configurations with fewer concentric rings ( $n$ equal to 2 and 3 ) to a wider truncation error percentile range to demonstrate consistency between all the considered electrode configurations that may allow estimation of optimal ranges of inter-ring distances for CREs with $n \geq 6$.

Author Contributions: Conceptualization, O.M.; methodology, O.M.; software, O.M., A.L. and A.B.; validation, O.M., A.L. and A.B.; formal analysis, O.M., A.L. and A.B.; writing-original draft preparation, O.M.; writing-review and editing, O.M.; visualization, O.M.; supervision, O.M.; project administration, O.M.; funding acquisition, O.M. All authors have read and agreed to the published version of the manuscript.

Funding: This research was funded by the National Science Foundation (NSF) Division of Human Resource Development (HRD) Tribal Colleges and Universities Program (TCUP), grant number 1914787 to O.M.

Acknowledgments: O.M. gratefully acknowledges Ernst Kussul and Tetyana Baydyk (National Autonomous University of Mexico, Mexico) for their constructive discussions and helpful comments. 
Conflicts of Interest: The authors declare no conflict of interest. The funders had no role in the design of the study; in the collection, analyses, or interpretation of data; in the writing of the manuscript, or in the decision to publish the results.

\section{References}

1. Wang, K.; Parekh, U.; Pailla, T.; Garudadri, H.; Gilja, V.; Ng, T.N. Stretchable Dry Electrodes with Concentric Ring Geometry for Enhancing Spatial Resolution in Electrophysiology. Adv. Healthc. Mater. 2017, 6, 1700552. [CrossRef] [PubMed]

2. Zena-Giménez, V.; Garcia-Casado, J.; Ye-Lin, Y.; Garcia-Breijo, E.; Prats-Boluda, G. A Flexible Multiring Concentric Electrode for Non-Invasive Identification of Intestinal Slow Waves. Sensors 2018, 18, 396. [CrossRef] [PubMed]

3. Prats-Boluda, G.; Ye-Lin, Y.; Pradas-Novella, F.; Garcia-Breijo, E.; Garcia-Casado, J. Textile Concentric Ring Electrodes: Influence of Position and Electrode Size on Cardiac Activity Monitoring. J. Sens. 2018, 2018, e7290867. [CrossRef]

4. Nasrollaholhosseini, S.H.; Mercier, J.; Fischer, G.; Besio, W. Electrode-Electrolyte Interface Modeling and Impedance Characterizing of Tripolar Concentric Ring Electrode. IEEE Trans. Biomed. Eng. 2019, 66, 2897-2905. [CrossRef] [PubMed]

5. Toole, C.; Martinez-Juárez, I.E.; Gaitanis, J.N.; Sunderam, S.; Ding, L.; DiCecco, J.; Besio, W.G. Source Localization of HighFrequency Activity in Tripolar Electroencephalography of Patients with Epilepsy. Epilepsy Behav. 2019, 101, 106519. [CrossRef] [PubMed]

6. Makeyev, O.; Musngi, M.; Moore, L.; Ye-Lin, Y.; Prats-Boluda, G.; Garcia-Casado, J. Validating the Comparison Framework for the Finite Dimensions Model of Concentric Ring Electrodes Using Human Electrocardiogram Data. Appl. Sci. 2019, 9, 4279. [CrossRef]

7. Garcia-Casado, J.; Ye-Lin, Y.; Prats-Boluda, G.; Makeyev, O. Evaluation of Bipolar, Tripolar, and Quadripolar Laplacian Estimates of Electrocardiogram via Concentric Ring Electrodes. Sensors 2019, 19, 3780. [CrossRef] [PubMed]

8. Aghaei-Lasboo, A.; Inoyama, K.; Fogarty, A.S.; Kuo, J.; Meador, K.J.; Walter, J.J.; Le, S.T.; Graber, K.D.; Razavi, B.; Fisher, R.S. Tripolar Concentric EEG Electrodes Reduce Noise. Clin. Neurophysiol. 2020, 131, 193-198. [CrossRef] [PubMed]

9. Liu, X.; Makeyev, O.; Besio, W. Improved Spatial Resolution of Electroencephalogram Using Tripolar Concentric Ring Electrode Sensors. J. Sens. 2020, 2020, 6269394. [CrossRef]

10. Estrada-Petrocelli, L.; Torres, A.; Sarlabous, L.; Rafols-de-Urquia, M.; Ye-Lin, Y.; Prats-Boluda, G.; Jane, R.; Garcia-Casado, J. Evaluation of Respiratory Muscle Activity by Means of Concentric Ring Electrodes. IEEE Trans. Biomed. Eng. 2021, 68, 1005-1014. [CrossRef] [PubMed]

11. Garcia-Casado, J.; Prats-Boluda, G.; Ye-Lin, Y.; Restrepo-Agudelo, S.; Perez-Giraldo, E.; Orozco-Duque, A. Evaluation of Swallowing Related Muscle Activity by Means of Concentric Ring Electrodes. Sensors 2020, 20, 5267. [CrossRef] [PubMed]

12. Makeyev, O. Solving the General Inter-Ring Distances Optimization Problem for Concentric Ring Electrodes to Improve Laplacian Estimation. Biomed. Eng. Online 2018, 17, 117. [CrossRef] [PubMed]

13. Makeyev, O.; Besio, W.G. Improving the Accuracy of Laplacian Estimation with Novel Variable Inter-Ring Distances Concentric Ring Electrodes. Sensors 2016, 16, 858. [CrossRef] [PubMed]

14. Makeyev, O.; Ding, Q.; Besio, W.G. Improving the Accuracy of Laplacian Estimation with Novel Multipolar Concentric Ring Electrodes. Measurement 2016, 80, 44-52. [CrossRef] [PubMed]

15. King, M.R.; Mody, N.A. Numerical and Statistical Methods for Bioengineering: Applications in MATLAB; Cambridge University Press: Cambridge, UK, 2010. 